\title{
Turismo social y turismo receptivo. Dinámicas competitivas y cooperativas a partir de la Nueva Ley de Turismo en Argentina ${ }^{1}$
}

\author{
Social tourism and inbound tourism. Competitive and cooperative dynamics \\ upon the New Tourism Law in Argentina
}

Recibido: 31 de enero de 2017 - Revisado: 09 de noviembre de 2017 - Aceptado: 08 de enero de 2018.

\section{Erica Schenkel ${ }^{2}$}

\begin{abstract}
Resumen
La investigación pretende echar luz en las dinámicas contrapuestas y cooperativas que impulsa la Nueva Ley de Turismo (25.997/05) en torno al impulso del turismo social y el fortalecimiento del turismo receptivo. A partir de la nueva normativa, el turismo es reconocido un derecho de las personas y en este marco se sientan las bases para el impulso de políticas de turismo social; al mismo tiempo que se destaca al turismo receptivo como actividad de exportación no tradicional, impulsando diferentes herramientas para su fortalecimiento. Más allá de esta complementariedad institucionalizada, la investigación comprueba que la importancia creciente que adquiere el turismo receptivo (en la afectación y ejecución de recursos, la creación de organismos específicos y la designación de personal), va en detrimento de la política de turismo social, que termina ocupando un lugar marginal en la agenda turística.
\end{abstract}

\section{Palabras clave}

Política turística, turismo social, turismo receptivo, Ley 25.997 de 2005, Argentina.

\begin{abstract}
The research analyzes the competitive and cooperative dynamics promoted by the New Tourism Law (25.997 / 05) related to the promotion of social tourism and the strengthening of receptive tourism. Within the new law, tourism is recognized as a right of the people and according to this framework, the foundations are laid for the promotion of social tourism policies. At the same time, receptive tourism is highlighted as a non-traditional export activity, promoting different tools for its endorsement. Although this institutionalized complementarity, research shows that the importance acquired by receptive tourism (in the execution of resources, the creation of specific organizations and the appointment of personal) goes against the social tourism policy, which ends up occupying a marginal place in the tourist agenda.
\end{abstract}

\section{Keywords}

Tourism policy, social tourism, inbound tourism, law 25.997 of 2005, Argentina.
${ }^{1}$ El artículo de investigación se enmarca dentro del proyecto: "Turismo y dinámicas territoriales: diferentes perspectivas de análisis" (24/G076), financiado por la Secretaría General de Ciencia y Tecnología de la Universidad Nacional del Sur, Argentina.

2 Docente - Investigadora en la Universidad Nacional del Sur, Buenos Aires, Argentina. Doctora en Ciencias Sociales con mención en Ciencias Políticas y Administración Pública. Becaria post-doctoral del Consejo Nacional de Investigaciones Científicas y Técnicas -CONICET.

Correo electrónico:

erica.schenkel@uns.edu.ar

(10) https://orcid.org/0000-0002-54562119

Para citar este artículo use: Schenkel, E. (2018). Turismo social y turismo receptivo. Dinámicas competitivas y cooperativas a partir de la Nueva Ley de Turismo en Argentina. Civilizar, 18(35), 39-52. doi:10.22518/usergioa/jour/ccsh/2018.2/a04 


\section{Introducción}

En el marco del proceso de internacionalización y crecimiento económico que sostienen la política turística argentina desde mediados de 1970 , el turismo pasa a ocupar un lugar central en la agenda pública a partir de las crisis del 2001, asociado a la necesidad de atraer divisas internacionales, ante la deficitaria balanza de pagos y la creciente deuda externa declarada en default. El Gobierno Nacional consolida una reformulación del turismo social como política pública, que complementa las virtudes distributivas, que históricamente fundamentaron su inserción en la agenda gubernamental, con otras económicas, vinculadas a la generación de riqueza y de empleo, que queda finalmente formalizada en la Nueva Ley Nacional de Turismo del año 2005.

La sanción de la Ley 25.997/05 y su Decreto Reglamentario ( $\left.\mathrm{N}^{\circ} 1.297 / 06\right)$, constituyen el hecho institucional de mayor relevancia en cuarenta años, al reemplazar la antigua Ley $14.574 / 58$, promulgada durante el gobierno del Dr. Arturo Frondizi. Dicha normativa reconoce al turismo como "un derecho social y económico de las personas dada su contribución al desarrollo integral en el aprovechamiento del tiempo libre y en la revalorización de la identidad cultural de las comunidades" (Ley 25.997, art. 2); destaca la necesidad de impulsar iniciativas de turismo social que permitan el ejercicio efectivo de estas prácticas, promoviendo los instrumentos necesarios para que el conjunto de la sociedad pueda acceder al ocio turístico en todas sus formas (Ley 25.997, Art. 38).

Paralelamente a dicho reconocimiento, la nueva norma proclama al turismo como una "actividad socioeconómica" de interés nacional, siendo ésta "prioritaria dentro de las políticas de Estado" (Ley 25.997, Art. 1, 3). En este marco, el turismo receptivo es distinguido como una "actividad de exportación" no tradicional y el sector privado como una "aliado estratégico" para su fortalecimiento, impulsando herramientas económicas similares a las que se otorgan a la actividad industrial, entre las que se destaca la creación de un organismo para la promoción en el mercado internacional, el Instituto Nacional de Promoción Turística -INPROTUR (Ley 25.997, Art. 1, 13).

La investigación busca responder cuáles son los impactos de la Nueva Ley una vez puestos en marcha dichos objetivos formulados. Particularmente pretende echar luz en las dinámicas competitivas y cooperativas que surgen asociadas al desarrollo de la política de turismo social y al fortalecimiento del turismo receptivo. Bajo este lineamiento pone en tensión: ¿Qué sucede con la política de turismo social una vez implementada? ¿La consolidación del turismo receptivo contribuye al desarrollo del turismo social o su impulso termina por limitarlo?

\section{Metodología}

La investigación presenta un estudio de tipo explicativo, que relaciona la política de turismo social que se formula en el periodo de post-crisis argentina del año 2001, con el fortalecimiento del turismo receptivo que sostiene la Ley 25.997/05, como actividad de exportación clave para el Estado. El estudio aporta diferentes lineamientos explicativos en torno a la consolidación del turismo receptivo y la posición secundaria que pasa a ocupar el turismo social entre las políticas del sector, transfiriendo una cantidad creciente de los recursos del área.

El estudio parte del análisis de políticas públicas, particularmente de los enfoques amplios de evaluación, que alejados de criterios deterministas, pretenden explicar la acción pública en el área y sus efectos en la sociedad, considerando la complejidad del análisis, el carácter intrínsecamente histórico de los procesos, la contextualización y la necesidad de abordajes holísticos, que se extiendan a los procedimientos y metodologías utilizadas, incluyendo técnicas cuantitativas y cualitativas de indagación (Oszlak y O’Donnell, 1995; Martínez, 2006; Pérez Sánchez, 2006). 
La investigación centra el análisis en la formulación de la política. En esta etapa se hacen visibles las decisiones de los actores político-administrativos a partir de programas y diferentes medidas administrativas, que son necesarias para su puesta en práctica y seguimiento (Mondragón, 2006).

Entre las técnicas de investigación, se destaca el análisis de documentos de tipo: normativos, Constituciones, Leyes, Decretos y Resoluciones; manifestaciones oficiales, Presupuestos Nacionales, Cuentas de Inversión, Memorias Detalladas del estado de la Nación, Cartas de Compromiso, Planes Estratégicos de Turismo Sustentable, discursos, escritos y prensa pública; y programas y planes especificos, difusión oficial e informes. La investigación documental se complementa con la realización de entrevistas abiertas a los principales actores ejecutores de la política de turismo social: el Director de Prestaciones Turísticas de la Nación y los Delegados Administrativos de los complejos turísticos estatales de Embalse y Chapadmalal.

\section{La formulación de la política pública: lineamientos para el análisis}

Las políticas constituyen respuestas institucionales a estados sociales considerados problemáticos, que se desarrollan en un contexto social, político, económico y tecnológico en permanente transformación. Los problemas públicos no existen en sí, sino que son construcciones artificiales que reflejan diferentes concepciones de la realidad (Gelambí, 2006; Ortega y Ruiz, 2006). Surgen de una lucha simbólica entre actores que constituidos a parir de diferentes naturalezas compiten entre sí por imponer su discurso a partir de sus valores e intereses. Subirats, Knoepfel, Larrue y Varone (2008), plantean que "un problema social se identifica y más tarde se 'tematiza' o se 'formatea' como problema público” (p. 127).

Una vez tematizado el problema social e incorporado en la agenda gubernamental, los actores político-administrativos formulan la política pública y hacen visibles sus decisiones mediante programas y una serie de medidas administrativas necesarias para su puesta en práctica y seguimiento. Knoepfel, Larrue, Varone e Hinojosa (2007) y Subirats et al., (2008) destacan que para el abordaje empírico de esta etapa, en primer lugar debe analizarse el programa de actuación político administrativo, como un producto clave. El mismo refiere al conjunto de normas y actos reglamentarios -leyes, decretos, acuerdos administrativos, órdenes de implementación y directrices administrativas- que los parlamentos, gobiernos o autoridades fijan para sustentar los objetivos, los instrumentos de intervención y las modalidades operativas de la acción pública. Constituyendo la base jurídica de la política, determina los derechos y obligaciones impuestos a los actores participantes, estableciendo las prioridades de orden cronológico, espacial y social.

El segundo producto que se identifica, es el acuerdo político-administrativo, la base organizativa y procedimental de la política (Knoepfel et al., y Subirats et al., 2008). Implica la designación de las autoridades y servicios administrativos que llevarán a cabo la implementación de la política pública, así como los recursos necesarios, que se materializarán en el presupuesto. Está formado por un conjunto de reglas institucionales formales e informales que regulan la atribución de funciones específicas, estableciendo una red de interacciones verticales y horizontales.

\section{Una breve contextualización histórica a la política de turismo social argentina}

Contemporáneamente a las primeras iniciativas europeas, el gobierno de Juan Domingo Perón cristalizó el turismo social como política distributiva, tendiente a disminuir la inequidad en el acceso al ocio entre las diferentes posiciones sociales. El Estado se 
convirtió en el principal promotor y articulador de este tipo de iniciativas, que, como en pocos países en el mundo, incluyó la construcción de complejos turísticos propios para los sectores sociales marginados. El turismo social se regularizó y extendió al conjunto del territorio nacional, destinando por primera vez financiación específica (Schenkel, 2015).

El Gobierno Nacional articuló una serie de acciones específicas tendientes a facilitar el acceso de los trabajadores al turismo y otras estructurales, que fueron determinantes al proveer tiempo de descanso y recursos financieros en los sectores sociales mayoritarios. La política de turismo social logró incorporar a las prácticas turísticas a sectores sociales que históricamente habían estado privados de su disfrute (Pastoriza y Torre, 1999).

En esta etapa el turismo social alcanzó una posición preponderante en la política turística argentina que perduró incluso hasta el último cuarto del siglo XX. Las extensas distancias con los principales centros turísticos emisivos, el escaso desarrollo tecnológico en el ámbito de los transportes y las comunicaciones y el retrasado proceso de democratización de las prácticas turísticas de algunos de los países vecinos, dejaron al turismo receptivo en un segundo plano, hasta fines de la década de 1970.

El periodo post-peronista circunscribe una posición itinerante del turismo social en la agenda gubernamental, asociada a la alta inestabilidad del sistema político. A partir de la invalidación de normas, la transferencia de recursos a provincias, sindicatos y privados, se desarticuló progresivamente el sistema estatal de turismo social, para nunca repetir el desarrollo alcanzado en los años 1950 (Schenkel, 2017).

La cancelación del histórico Fondo de Turismo Social a fines de los 1970 por decisión de la última dictadura militar en Argentina, implicó un quebranto irremediable en el desarrollo del turismo social, que afectó incluso el mante- nimiento de los complejos turísticos construidos en el peronismo, representando el fin del periodo que había circunscripto el origen y la consolidación de la cuestión como política pública a mediados del siglo XX.

A pesar de que el restablecimiento del orden democrático en el año 1983 significó la reincorporación del turismo social en la agenda gubernamental, la importancia creciente que adquiere el turismo receptivo, asociado a necesidades de internacionalización y crecimiento económico del Estado, hacen que comience a ceder participación a favor de esta política receptiva, transfiriendo una cantidad creciente de los recursos del área. En este periodo, los decisores públicos inician una reconversión del turismo social como política pública, que amplió la participación a actores privados, concesionarios y hoteleros, planteó objetivos económicos asociados al crecimiento y la competitividad del sector e identificó nuevos grupos de destinatarios.

\section{La política de turismo social post-crisis argentina del 2001}

El turismo pasa a ocupar un lugar central en la agenda pública post-crisis del 2001, tendiente a atraer las necesarias divisas internacionales, ante la deficitaria balanza de pagos y la abultada deuda externa declarada en default ${ }^{1}$. La política turística es considerada un instrumento central del Gobierno Nacional para la ansiada reactivación económica.

En este marco se pone en marcha una activa política en el área, estructurada con base en los beneficios económicos que genera la actividad, el fortalecimiento institucional, las inversiones en infraestructura necesarias y la cooperación con el empresariado turístico. El turismo pasa a formar parte de las doce políticas de Estado que define el gobierno para impulsar el superávit, la recaudación, la inversión, la infraestructura y el crecimiento económico (Presidencia de la Nación, 2003; Jefatura de 
Gabinete de Ministros, 2006a). Específicamente en materia turística, la entonces Secretaría de Turismo debía "desarrollar la Actividad Turística como motor de la Economía" (Jefatura de Gabinete de Ministros -JGM, 2006b, p. 297).

Esta fundamentación económica que pasa a sostener al turismo en la agenda gubernamental, queda de manifiesto en la adscripción y jerarquía que adquiere el organismo específico. La histórica Secretaría de Turismo de la Nación, en primera instancia, pasa al Ministerio de Producción, que reúne aquellas áreas con "fuerte sesgo productivo": agricultura, ganadería y pesca, industria, turismo y comercio exterior (Decreto 2025/08); posteriormente al Ministerio de Industria (Decretos 1365/09 y 1366/09), luego denominado Ministerio de Industria y Turismo (Decreto 1458/09), que integra turismo con las áreas de industria y comercio exterior; finalmente, se convierte en un órgano autónomo por primera vez en la historia argentina, como Ministerio Nacional de Turismo (Decretos 919/10 y 921/10), constituyendo el decimocuarto ministerio del Estado. El decreto destaca la trascendencia que el turismo representa como "sector de desarrollo alternativo de la actividad económica" permitiendo la generación de empleo, de divisas y la reconversión de las economías regionales, y contribuyendo al desarrollo de la economía nacional y de la población en su conjunto.

Estos pronunciamientos quedan materializados en la sanción de la Nueva Ley Nacional de Turismo (2005). El turismo deja de ser institucionalizado como mera actividad de ocio y recreación (Ley 14.574/58) para ser proclamado como "actividad socioeconómica, estratégica y esencial", siendo ésta "prioritaria dentro de las políticas de Estado" (Ley 25.997, Art. 1, 3). El turismo receptivo pasa a distinguirse como una "actividad de exportación" no tradicional y el sector privado como una "aliado estratégico" para su desarrollo, impulsando herramientas económicas similares a las que se otorgan a la actividad industrial, entre las que se destaca la creación del INPROTUR, destinado a desarrollar programas, planes y estrategias para la promoción de los arribos internacionales.

El Instituto Nacional de Promoción Turística se convierte en el único organismo del área con financiación específica por ley: aportes de la Nación, el $40 \%$ del impuesto a los pasajes aéreos, fluviales y marítimos al exterior, subsidios, legados, cesiones, herencias o donaciones, aportes del sector privado, ingresos derivados de la realización de conferencias, seminarios, cursos y publicaciones, rentas, usufructos e intereses de sus bienes, ingresos provenientes de impuestos nacionales que pudieran crearse y otros fondos acorde al carácter legal y a las finalidades.

Partiendo de la premisa de transformar el turismo de pocos en un turismo para todos, la entonces Secretaría de Turismo (2003) sostiene que el turismo receptivo logrará su pleno sentido "solo" cuando la totalidad de los "compatriotas" accedan al disfrute de estas prácticas, a partir de una activa política de turismo social que consolide la "demanda de turismo interno" y atienda a los "sectores de menores recursos", como "respuesta al requerimiento social en suspenso y contenido en periodos anteriores", superando factores económicos, sociales y culturales, definiendo al turismo social una "política turística inclusiva" (Secretaría de Turismo de la Nación -SECTUR, 2007).

En esta línea, la nueva Ley Nacional de Turismo lo define "un derecho social y económico de las personas" y destina un título específico al turismo social (V), así como su Decreto Reglamentario (IV), definiéndolo como "aquellos instrumentos y medios que otorguen facilidades para que todos los sectores de la sociedad puedan acceder al ocio turístico en todas sus formas, en condiciones adecuadas de economía, seguridad y comodidad" (Ley 25.997/05, Art. 38).

La norma insta a la entonces Secretaría a elaborar programas de turismo social, que pro- 
muevan la prestación de servicios accesibles a la población mediante la operación de los complejos turísticos de su dependencia y la suscripción de acuerdos con prestadores de servicios turísticos, organizaciones sociales y empresas privadas (Ley 25.997/05; Decreto 919/2010).

Sostiene que las tarifas deben facilitar el acceso al turismo a los "sectores de la sociedad con menos disponibilidades o recursos", constatar que los servicios prestados cumplan con los estándares de calidad, fomentar los destinos no tradicionales y emergentes, apoyar a organismos públicos y privados vinculados con las políticas sociales y a las pequeñas y medianas empresas proveedoras de servicios turísticos y contribuir al diseño de instrumentos innovadores que posibiliten incluir a todos los sectores al acceso al turismo (Decreto 1.297/06, Art. 32).

Esta reformulación del turismo social complementa las históricas causas sociales que explican la temprana incorporación de la cuestión entre las políticas públicas nacionales, en el Primer Peronismo a mediados del siglo XX; con otras económicas, tendientes a fortalecer el turismo doméstico, incrementar los ingresos y empleos del sector, impulsar la inversión turística pública y privada y contribuir a la estabilidad del empresariado turístico (Figura 1).
El turismo social pasa a definirse: "como un vehículo de desarrollo económico y social tendiente a contrarrestar la estacionalidad, redistribuir la demanda interna y asegurar el ejercicio del derecho al Turismo a toda la población" (SECTUR, 2005). El Director de Prestaciones Turísticas afirma que estas nuevas finalidades económicas asociadas al desarrollo del turismo social se deben buscar junto a las tradicionales causas sociales: "el gran desafío es poder complementar el factor reparador de turismo social para el disfrute de ocio y el factor económico esencial para el Estado Nacional" (Comunicación personal, entrevista a Director, 2014).

Marcando una continuidad con la política del primer Peronismo, el gobierno sostiene que la reactivación de este tipo de iniciativas significan la actualización y puesta en práctica del "concepto primigenio de Turismo Social del justicialismo" como vehículo para lograr una mayor equidad, bienestar y crecimiento de la ciudadanía excluida: "estamos convencidos que abrevando en sus fuentes afianzaremos el turismo social en forma definitiva partiendo del ejercicio pleno de un derecho" (SECTUR, 2003; Mintur, 2010). En este punto se destaca la importancia de emprender instrumentos concretos, a fin de aprovechar la manifiesta volun-

Figura 1.

Reformulación del turismo social post-crisis del 2001

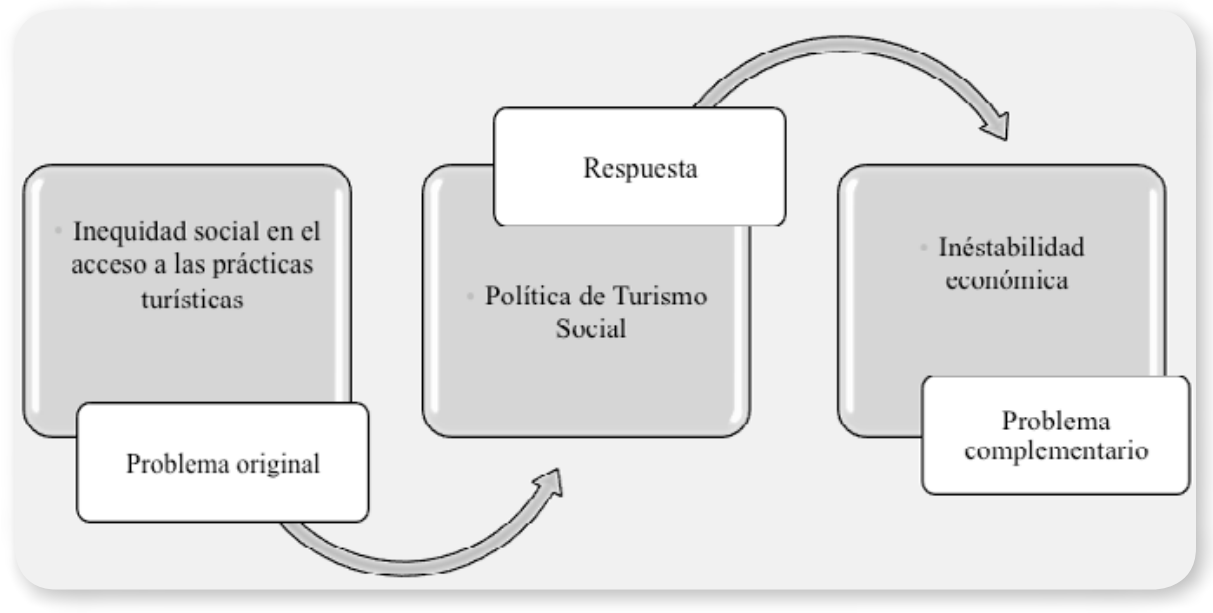

Fuente: Elaboración propia. 
tad política para llevarlos a cabo, "quizá como ningún otro momento de la Argentina en las últimas décadas" (SECTUR, 2003; Jefatura de Gabinete de Ministros -JGM, 2004), marcando un antes y un después con los distintos gobiernos sucedidos desde el último golpe de Estado.

El Gobierno Nacional inicia un proceso de recuperación de los complejos de Embalse (Córdoba) y Chapadmalal (Buenos Aires) ${ }^{2}$, que logra incorporar cerca de 2.000 plazas al sistema de turismo social argentino. Con recursos del propio Ministerio de Turismo, de los concesionarios y del Ministerio de Desarrollo Social con el Programa Argentina Trabaja, se logra la reapertura de hoteles, polideportivos y áreas recreativas, la recuperación de infraestructura, equipamiento e instalaciones y la refuncionalización de espacios en desuso (Planillas de observación, 2015; Comunicación personal, entrevistas a delgados administrativos, 2015).

En este marco se lleva a cabo un relanzamiento de los Programas de Turismo Social, destinados principalmente a la población de escasos recursos de todo el país, siendo su objetivo brindarle la posibilidad de poder contar con un período de vacaciones (SECTUR, 2004, 2009; Mintur, 2014). Las prestaciones se estructuran a partir del tradicional Programa en Unidades Turísticas, con base en los complejos estatales de Chapadmalal y Embalse, que continua con el sistema de concesiones que había sido implementado por los últimos gobiernos, y el Programa Federal de Turismo Social (2000), que surge en vísperas de la crisis, tendiente a combatir la estacionalidad de los destinos maduros, articulando con hoteles categoría 1, 2 y 3 estrellas en diferentes provincias del país. El entonces Presidente de la República destacaba en el Mensaje de Apertura del año legislativo que el desarrollo del turismo social se emprende como "vehículo para lograr la posibilidad de acceso al turismo a todos los sectores de la población" (Presidencia de la Nación, 1 de marzo de 2006).
La gestión de los Programas quedó a cargo de la Dirección de Prestaciones Turísticas (creada a mediados de los 90), dependiente de la entonces Secretaría de Turismo, luego del Ministerio, destinada a fomentar las prácticas turísticas en "grupos vulnerables", mediante la implementación de las prestaciones ofrecidas, el ejercicio del control de calidad y la facilitación de la accesibilidad turística. A partir del año 2004, la Dirección pasa a depender directamente de la Subsecretaría de Turismo, luego, con la creación del Ministerio, de la Secretaría, dejando el área de Desarrollo y Promoción Turística, a la que adscribió hasta entonces. Junto a Relaciones Internacionales, constituyen las únicas Direcciones, de las once existentes a mediados del año 2014, que tienen vinculación directa con el organismo decisional.

\section{El turismo social a partir de la implementación de la Nueva Ley Nacional de Turismo}

La importancia del turismo social en la política sectorial y nacional comienza a diluirse a medida que avanza la implementación de la Ley 25.997 de 2005, sus fundamentos y organismos vinculantes. Al analizar diferentes elementos asociados a la política, como el crédito afectado y ejecutado, los recursos humanos asignados y la cantidad y tipos de beneficiarios, se observa que el turismo social no sólo no fortalece su participación desde entonces sino que la misma se ve claramente disminuida.

En cuanto a los recursos asignados, la Dirección de Prestaciones cuenta con crédito específico en los diferentes presupuestos nacionales $^{3}$. El mismo se distribuye entre la administración de las Unidades Turísticas, las prestaciones turísticas en los complejos estatales y el Turismo Social Federal, sucesor del Programa Jubilados y Pensionados, que en la década de 1990 centralizaba la tercera parte del presupuesto. Los principales gastos corresponden a servicio técnico y profesionales, seguidos de personal y luego, bienes de consumo, manteni- 
miento, reparación y limpieza, bienes de uso, viáticos y servicio comerciales (Leyes de Presupuesto, 1994-2014).

La evolución refleja como los créditos afectados al turismo social crecen su participación en los primeros años post-crisis, para disminuir a partir del año 2005, momento que es sancionada la Ley de Turismo 25.997, y comienzan a implementarse los nuevos lineamientos destinados a consolidar al turismo como actividad económica y al turismo receptivo como área clave. Esta disminución se acentúa incluso con la creación del Ministerio (2010), que reafirma las necesidades económicas asociadas al desarrollo de la actividad. Mientras que la Administración de Turismo aumenta su participación en el presupuesto público en ambas ocasiones, el turismo social la disminuye marcadamente, estabilizándose en torno al 13\% del gasto turístico. Esta participación en el área está muy por debajo de la que supo alcanzar en la década del noventa, cuando el turismo social continuaba ocupando una posición destacada entre las políticas turísticas, concentrando más del 55\% del crédito en turismo (Figura 2).

Figura 2.

Recursos destinados al turismo social en función de la Administración Central en general y el área de Turismo en particular (1994-2014)

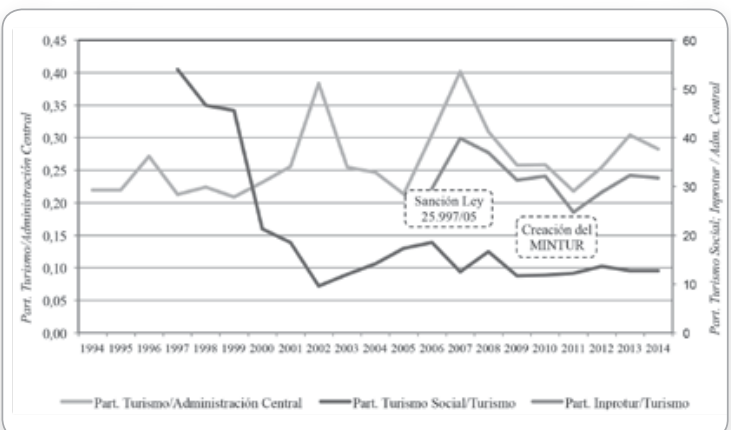

Fuente: Elaboración propia con base en Leyes de Presupuesto (1994-2014).

Esta retracción se relaciona a la complejización que adquiere la Administración de Turismo en la última década, ampliando y diversificando sus departamentos, actividades, programas y planes, en función de la institucionalización del turismo como actividad económica. Al comparar la nueva estructura organizacional con la del año 1996, cuando el turismo social alcanzó el máximo de los recursos afectados, se observan once nuevos organismos, cuatro Subsecretarías -Coordinación, Calidad Turística, Desarrollo y Promoción Turística- y siete Direcciones -Sumarios, Recursos Humanos y Organización, Sistemas Informáticos, Relaciones Internacionales, Agencias de Viajes y Turismo, Calidad Turística y Desarrollo Turístico-, que pasan a competir con el presupuesto asignado al turismo social (Decretos 1407/96 y 1067/13). Entre estos organismos se destaca la creación del Instituto Nacional de Promoción Turística (2006), como ente descentralizado, que concentra cerca del $40 \%$ de los recursos con los que contaba la entonces Secretaría ${ }^{4}$.

En cuanto a los recursos ejecutados, del mismo modo que los presupuestados, lejos de potenciarse con la sanción de la Ley de Turismo (2005) y la creación del MINTUR (2010), ambos hechos ocasionan una significativa caída (Figura 3). Es dable destacar que estos créditos destinados al turismo social quedan otra vez marcadamente por debajo de la década pasada, que alcanzan un máximo en 1997 con el 80\% del gasto turístico.

Figura 3.

\section{Participación del gasto en turismo social en función del total del área}

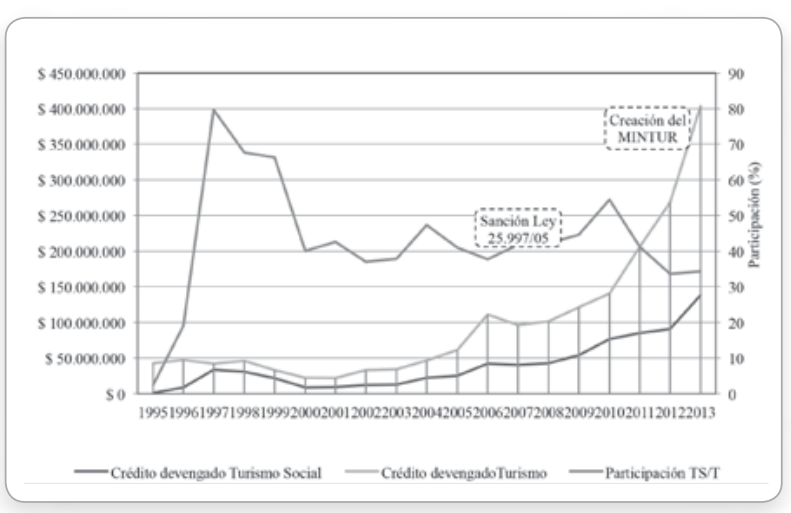

Fuente: Elaboración propia con base en las Cuentas de Inversión (1995-2013). 
Similar comportamiento presentan los recursos humanos afectados. A medida que se implementa la reorganización de la SECTUR (Ley 25.997/05), ampliando y diversificando sus departamentos, actividades y organismos, disminuye la participación del turismo social en el área, mientras que ésta crece personal. Para el año 2014, el Ministerio de Turismo reúne cerca de 500 empleados, entre permanentes, transitorios y contratados, duplicando la planta del año 2000; mientras que los complejos destinados al turismo social descienden a cerca de $100 \mathrm{em}$ pleados por Unidad, cercano a los niveles mínimos de la crisis del 2001 (Figura 4).

Figura 4.

Recursos humanos afectados al MINTUR y a las unidades turísticas

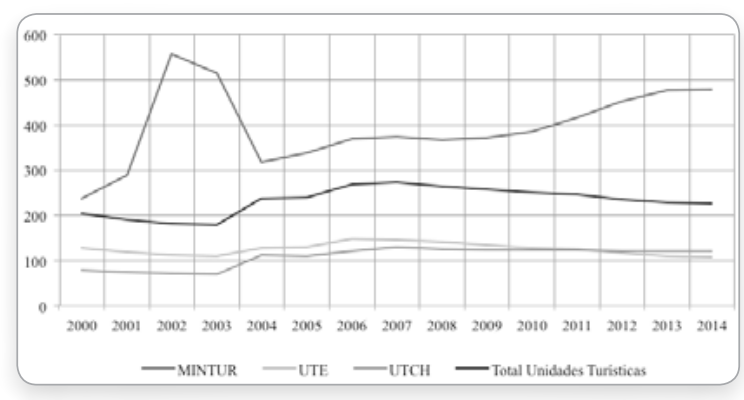

Fuente: Elaboración propia a partir de información provista por Dirección de Recursos Humanos (2014).

Estos reajustes se traducen en la cantidad de beneficiarios que acceden al turismo social a lo largo del periodo. Luego de alcanzar valores mínimos en plena crisis del año 2001, las prestaciones crecen hasta el año 2007, con un aumento superior al $400 \%$, para luego caer y estabilizarse en valores de la década del 90, en torno a los 150.000 turistas anuales (Figura 5).

En cuanto al tipo de beneficiario, en el año 2003, de cada diez personas que accedían a los Programas, cinco correspondían al colectivo de jubilados, tres a escolares y dos a familias; diez años después, cinco pertenecen a familias, tres a escolares y dos a jubilados (Dirección de Prestaciones Turísticas, 2014b). En el caso del Plan Eventos, aparecen resultados en las Memorias Detalladas del estado de la Nación de los ejercicios 2004: 17.674 participantes en Embal- se y 7.248 en Chapadmalal; y 2005: 17.864 y 34.703 , respectivamente. Si se comparan estas cifras con la totalidad de las personas beneficiarias de los Planes tradicionales, Escolar, Tercera Edad y Familiar, representan una cantidad significativa, alcanzando el $25 \%$ en el año 2004 y el $45 \%$ en el año 2005.

Figura 5.

Cantidad de personas que acceden a los Programas

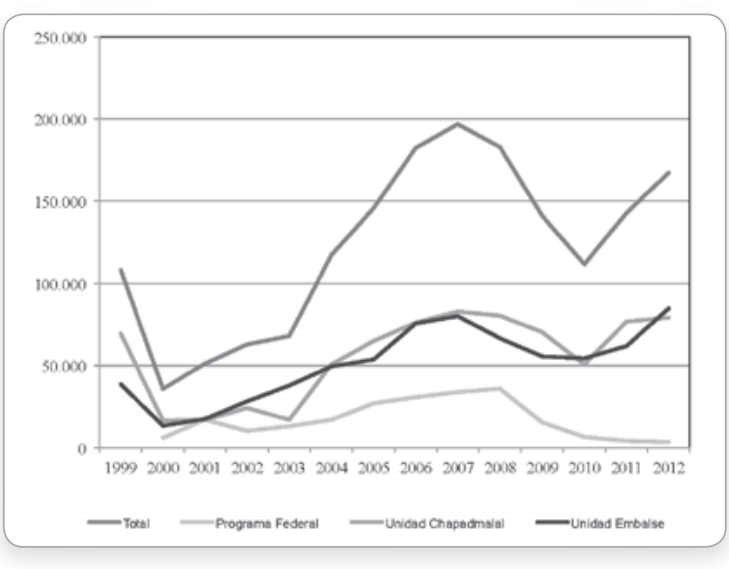

Fuente: Elaboración propia a partir de registros de la DPT $(2014 b)^{*}$.

* La cantidad de beneficiarios constituye una aproximación en función de una media de 6 días por turista para Unidades Turísticas y 5 para Federal.

Estos grupos de destinatarios presentan una línea de continuidad con la política implementada en las últimas décadas, cuando el turismo social dejó de asistir a los sectores obreros, para dirigirse a distintos colectivos, como familias numerosas, jóvenes, tercera edad, personas con discapacidad y grupos de escolares, dando cuenta de las estructuras sociales dinámicas y fragmentadas que priman en las sociedades contemporáneas y de las nuevas finalidades asociadas al turismo social. La representación del universo de beneficiarios deja de centralizarse en los sindicatos, que pasan a integrar una compleja red de naturaleza pública y privada, en la cual intervienen otros participantes, principalmente organizaciones sin fines de lucro, movimientos sociales y municipios, que asumen la representatividad de estos nuevos colectivos de usuarios. 


\section{Conclusiones}

El análisis comprueba que a pesar de los principios de complementariedad que sostienen la nueva normativa, la importancia creciente que le adjudica al turismo como actividad económica $\mathrm{y}$ al turismo receptivo como política clave del sector (asociada a necesidades de internacionalización y de crecimiento económico del Estado), terminan por relegar el turismo social en pos del fomento de los arribos internacionales.

En el periodo de post-crisis del año 2001, ante un Gobierno Nacional que se reposiciona como actor clave en el área, la entonces Secretaría de Turismo, formula una política específica de turismo social, que complementa los históricos fundamentos sociales que dieron origen a la cuestión, con otros económicos, a fin de reimpulsar las prestaciones turísticas inmersas en un profundo retroceso. El turismo social se presenta así como una continuidad de la política implementada en el Primer Peronismo en los años 50, significando la "actualización" y "puesta en práctica" del concepto primigenio justicialista, tendiente a alcanzar mayor equidad, bienestar y crecimiento de la ciudadanía excluida (SECTUR, 2007).

Esta retórica asociada a los primeros gobiernos de Perón, es acompañada de avances concretos en la sanción de normativa específica y en la infraestructura de los complejos vacacionales. En cuanto al primero, el derecho a las vacaciones anuales pagadas generalizado en el Primer Peronismo (Decreto 1740/45), que en principio estaba circunscripto al sector trabajador y a partir de la Reforma de 1994 se amplía al conjunto de la población, se consolidó en el año 2005, con la proclamación del turismo como derecho económico y social de las personas, destacando la necesidad de impulsar iniciativas de turismo social, que permitan el ejercicio efectivo a estas prácticas, para que el conjunto de la sociedad pueda acceder al ocio turístico en todas sus formas.
En cuanto a los complejos, a fin de reivindicar el "espíritu" con el que fueron creados los complejos de Chapadmalal y Embalse en los años 50, el Gobierno Nacional inició un significativo proceso de recuperación de las Unidades, que posibilita la reapertura de hoteles, polideportivos y áreas recreativas, la recuperación de infraestructura, equipamiento e instalaciones y la refuncionalización de espacios en desuso, incluyendo el relanzamiento de los históricos torneos Evita. Luego de treinta años de retracción, se incorporaron 2.000 plazas al sistema estatal de turismo social, aumentando las prestaciones turísticas en ambas Unidades.

Estos significativos avances impulsados desde las áreas decisionales, contrastan con el sistema de turismo social que se presenta prácticamente inalterable. A pesar de la retórica oficial, que afirma recuperar el turismo social del Primer Peronismo luego de décadas de abandono, expresando una continuidad con lo llevado a cabo en los años $50 \mathrm{y}$, al mismo tiempo, una ruptura con la política implementada en el último cuarto de siglo; la política de post-crisis, consolida el proceso de reformulación que se desarrolla desde el regreso de la democracia. El órgano ejecutor creado a mediados de la década de los 90, incluso las autoridades a cargo del área; los programas y planes, estructurados en un sistema de subvenciones y acuerdos de precios, en servicios de estadía y alimentación, destinados a colectivos de tercera edad, familias, escolares y personas con discapacidad; la cantidad de prestaciones ejecutadas y de personas beneficiadas; los actores relevantes que participan del sistema, a partir de concesionarios y hoteleros; los fundamentos que justifican la acción en el área, así como los recursos asignados; sin presentar cambios sustanciales, constituyen una extensión de la acción pública implementada en las últimas décadas.

Como sucede con las demás políticas turísticas, el turismo social se implementa subsidiario al turismo receptivo, prioritario en la agenda gubernamental como "actividad de 
exportación no tradicional", que pasa a concentrar una cantidad creciente de los recursos del área (económicos y humanos), así como la producción de información específica y los controles de seguimiento. La creación del Ministerio, que reafirma estas finalidades económicas asociadas al desarrollo de la actividad, lejos de fortalecer al turismo social, acentúa su posición marginal en la arena turística. La normativa formulada, la afectación y ejecución de recursos, la creación de organismos específicos y la designación de personal, revela la prioridad gubernamental, tendiente a consolidar los arribos internacionales, ante una deficitaria balanza de pagos en crecimiento.

\section{Referencias}

Ferrer, A. (2010). El futuro de nuestro pasado. La economía argentina en su segundo centenario. Buenos Aires: Fondo de Cultura Económica.

Gelambí Torrel, M. (2006). La terminación de políticas públicas. En M. Pérez Sánchez, (Ed.). Análisis de Políticas Públicas, (pp. 197-220). Granada: Universidad de Granada.

Jefatura de Gabinete de Ministros - [JGM]. (2004). Memoria detallada del estado de la Nación del año 2003. Autor: Argentina.

Jefatura de Gabinete de Ministros. - [JGM]. (2006a). El turismo como política de Estado en la República Argentina. Buenos Aires: Observatorio de Políticas Públicas.

Jefatura de Gabinete de Ministros - [JGM]. (2006b). Memoria detallada del estado de la Nación del año 2005. Buenos Aires: Autor.

Knoepfel, P., Larrue, C., Varone, F., y Hinojosa, M. (2007). Hacia un modelo de análisis de políticas públicas operativo. Un enfoque basado en los actores, sus recursos y las instituciones. Ciencia Política, 2(3), 6-29.
Martínez del Olmo, M. (2006). La evaluación de políticas y programas públicos. En M. Pérez Sánchez (Ed.). Análisis de Politicas Públicas (pp. 175-195). Granada: Universidad de Granada.

Ministerio de Turismo de la Nación - [MINTUR]. (2010). Dirección de Prestaciones Turísticas. Turismo Inclusivo - Turismo para Todos. Buenos Aires: Mintur.

Ministerio de Turismo de la Nación-[MINTUR]. (2014). Programas de Turismo Social. [En línea]. Recuperado de http://www.turismo.gov.ar/indexfs.html [Consulta: 4 de marzo de 2014]

Mondragón, J. (2006). El proceso de toma de decisiones públicas: de la formulación de la decisión a la asignación de recursos. En M. Pérez Sánchez (Ed.), Análisis de Politicas Públicas (pp. 134-152). Granada: Universidad de Granada.

Ortega Pérez, N., y Ruiz Seisdedos, S. (2006). Definición de problemas y diseño de la agenda. En M. Pérez Sánchez (Ed.), Análisis de Políticas Públicas (pp. 111-131). Granada: Universidad de Granada.

Oszlak, O., y O’Donnell, G. (1995). Estado y políticas estatales en América Latina: Hacia una estrategia de investigación. Redes, 2(4), 99-128.

Pastoriza, E., y Torre, J. (1999). Mar del Plata, un sueño de los argentinos. En F. Devoto y M. Madero (Dirs.), Historia de la vida privada en la Argentina. La Argentina entre multitudes y soledades. De los años treinta a la actualidad (pp. 48-77). Buenos Aires: Taurus.

Pérez Sánchez, M. (2006). El Estudio de las Políticas Públicas. En autor (Ed.), Análisis de Politicas Públicas (pp. 51-75). Granada: Universidad de Granada.

Presidencia de la Nación. (2003). Políticas de Estado. Buenos Aires: Autor. 
Presidencia de la Nación. (1 de marzo de 2006). Mensaje de Apertura ante la Asamblea Legislativa. Buenos Aires: Autor.

República de Argentina. Constitución de la Nación Argentina (1994). Reforma de 1994. Buenos Aires.

República de Argentina. Ley de Presupuesto de 1995. Oficina Nacional de Presupuesto MECON. Secretaría de Hacienda.

República de Argentina. Ley de Presupuesto de 1996. Oficina Nacional de Presupuesto MECON. Secretaría de Hacienda.

República de Argentina. Ley de Presupuesto de 1997. Oficina Nacional de Presupuesto MECON. Secretaría de Hacienda.

República de Argentina. Ley de Presupuesto de 1998. Oficina Nacional de Presupuesto MECON. Secretaría de Hacienda.

República de Argentina. Ley de Presupuesto de 1999. Oficina Nacional de Presupuesto MECON. Secretaría de Hacienda.

República de Argentina. Ley de Presupuesto de 2000. Oficina Nacional de Presupuesto MECON. Secretaría de Hacienda.

República de Argentina. Ley de Presupuesto de 2001. Oficina Nacional de Presupuesto MECON. Secretaría de Hacienda.

República de Argentina. Ley de Presupuesto de 2002. Oficina Nacional de Presupuesto MECON. Secretaría de Hacienda.

República de Argentina. Ley de Presupuesto de 2003. Oficina Nacional de Presupuesto MECON. Secretaría de Hacienda.

República de Argentina. Ley de Presupuesto de 2004. Oficina Nacional de Presupuesto MECON. Secretaría de Hacienda.

República de Argentina. Ley de Presupuesto de 2005. Oficina Nacional de Presupuesto MECON. Secretaría de Hacienda.
República de Argentina. Ley de Presupuesto de 2006. Oficina Nacional de Presupuesto MECON. Secretaría de Hacienda.

República de Argentina. Ley de Presupuesto de 2007. Oficina Nacional de Presupuesto MECON. Secretaría de Hacienda.

República de Argentina. Ley de Presupuesto de 2008. Oficina Nacional de Presupuesto MECON. Secretaría de Hacienda.

República de Argentina. Ley de Presupuesto de 2009. Oficina Nacional de Presupuesto MECON. Secretaría de Hacienda

República de Argentina. Ley de Presupuesto de 2010. Oficina Nacional de Presupuesto MECON. Secretaría de Hacienda.

República de Argentina. Ley de Presupuesto de 2011. Oficina Nacional de Presupuesto MECON. Secretaría de Hacienda.

República de Argentina. Ley de Presupuesto de 2012. Oficina Nacional de Presupuesto MECON. Secretaría de Hacienda.

República de Argentina. Ley de Presupuesto de 2013. Oficina Nacional de Presupuesto MECON. Secretaría de Hacienda.

República de Argentina. Ley de Presupuesto de 2014. Oficina Nacional de Presupuesto MECON. Secretaría de Hacienda.

República de Argentina. Ley Nacional $\mathrm{N}^{\circ}$ 14.574 de 1958. Boletín Oficial de la República Argentina. Buenos Aires, 11 de noviembre de 1958.

República de Argentina. Ley Nacional $\mathrm{N}^{\circ}$ 25.997 de 2005. Boletín Oficial de la República Argentina. Buenos Aires, 7 de enero de 2005.

República de Argentina. Decreto $\mathrm{N}^{\circ} 1.297$ de 2006. Boletín Oficial de la República Argentina. Buenos Aires, 29 de septiembre de 2006. 
República de Argentina. Decreto $\mathrm{N}^{\circ} 2.025$ de 2008. Boletín Oficial de la República Argentina. Buenos Aires, 26 de noviembre de 2008.

República de Argentina. Decreto Nº 1.365 de 2009. Boletín Oficial de la República Argentina. Buenos Aires, 2 de octubre de 2009.

República de Argentina. Decreto № 1.366 de 2009. Boletín Oficial de la República Argentina. Buenos Aires, 2 de octubre de 2009.

República de Argentina. Decreto $\mathrm{N}^{\circ} 1.458$ de 2009. Boletín Oficial de la República Argentina. Buenos Aires, 13 de octubre de 2009.

República de Argentina. Decreto $N^{\circ} 919$ de 10. Boletín Oficial de la República Argentina. Buenos Aires, 30 de junio de 2010.

República de Argentina. Decreto $\mathrm{N}^{\circ} 921$ de 2010. Boletín Oficial de la República Argentina. Buenos Aires, 30 de junio de 2010.

República de Argentina. Decreto $N^{\circ} 1.067$ de 2013. Boletín Oficial de la República Argentina. Buenos Aires, 7 de agosto de 2013.

Schenkel, E. (2014). La inserción del turismo social a la agenda pública en la Argentina. Papers de Turisme, (56), 1-19.

Schenkel, E. (2017). La desarticulación de la política de turismo social argentina de mediados de siglo X. Anais Brasileiros de Estudos Turísticos, 7(2), 50 - 64.

Secretaría de Turismo de la Nación - [SECTUR]. (2003). Turismo Social en la Argentina. Turismo para todos. Buenos Aires: Autor.

Secretaría de Turismo de la Nación - [SECTUR]. (2004). Primera Carta Compromiso con el Ciudadano. Programa Carta Compromiso con el Ciudadano. Secretaría de Gabinete y Gestión Pública.
Secretaría de Turismo de la Nación - [SECTUR]. (2005). Turismo 2016. Plan Federal Estratégico de Turismo Sustentable. Autor.

Secretaría de Turismo de la Nación - [SECTUR]. (2007). Turismo Social. En 100 Años de Turismo Argentino. Autor.

Secretaría de Turismo de la Nación - [SECTUR]. (2009). Segunda Carta Compromiso con el Ciudadano. Programa Carta Compromiso con el Ciudadano. Secretaría de Gabinete y Gestión Pública.

Subirats, J., Knoepfel, P., Larrue, C., y Varone, F. (2008). Análisis de políticas públicas y gestión pública. Barcelona: Ariel.

\section{Notas}

1 Los crecientes déficits fiscales y comerciales, el aumento sostenido de la deuda externa y la fuga de capitales, confluyeron en la peor crisis de la historia económica argentina, que eclosionó en diciembre del año 2001, con los consecuentes impactos sociales y políticos (Ferrer, 2010).

2 Ambos complejos habían sido construidas por el Primer Peronismo a mitad del siglo XX. La quita del Fondo de Turismo Social en la década del 70 había iniciado un proceso de deterioro de los complejos que los encuentra en plena crisis del 2001, con la mayoría de los establecimientos clausurados, evaluando proyectos de transferencia y privatizaciones (Schenkel, 2017).

3 Dentro de la Jurisdicción de Presidencia de la Nación hasta el año 2008, Ministerio de Producción en el año 2009, Ministerio de Industria y Turismo en el año 2010 y desde entonces, dentro del Ministerio de Turismo.

4 Si bien el INPROTUR, como todo organismo descentralizado presenta presupuesto propio a fines comparativos se asocia al presupuesto del área de Turismo. 
